\title{
Beyond the Goldenberg-Vaidman protocol: Secure and efficient quantum communication using arbitrary, orthogonal, multi-particle quantum states
}

\author{
Chitra Shukla ${ }^{1}$, Anirban Pathak ${ }^{1,2}$ and R. Srikanth ${ }^{3,4}$ \\ July 3, 2018 \\ ${ }^{1}$ Jaypee Institute of Information Technology, A-10, Sector-62, Noida, India. \\ ${ }^{2}$ RCPTM, Joint Laboratory of Optics of Palacky University and
}

'Institute of Physics of Academy of Science of the Czech Republic, Faculty of Science, Palacky University, 17. listopadu 12, 77207 Olomouc, Czech Republic.

\author{
${ }^{3}$ Poornaprajna Institute of Scientific Research, Sadashivnagar, Bengaluru- 560080, India. \\ ${ }^{4}$ Raman Research Institute, Sadashivnagar, Bengaluru- 560060, India.
}

\begin{abstract}
It is shown that maximally efficient protocols for secure direct quantum communications can be constructed using any arbitrary orthogonal basis. This establishes that no set of quantum states (e.g. GHZ states, $W$ states, Brown states or Cluster states) has an advantage over the others, barring the relative difficulty in physical implementation. The work provides a wide choice of states for experimental realization of direct secure quantum communication protocols. We have also shown that this protocol can be generalized to a completely orthogonal state based protocol of Goldenberg-Vaidman (GV) type. The security of these protocols essentially arises from duality and monogamy of entanglement. This stands in contrast to protocols that employ non-orthogonal states, like Bennett-Brassard 1984 (BB84), where the security essentially comes from non-commutativity in the observable algebra.
\end{abstract}

K'eywords: DSQC, QSDC, QKD

PACS: 03.67.Dd,03.67.Hk, 03.65.Ud

N

\section{Introduction}

Since the proposal for the first protocol for quantum key distribution [1, and the other early QKD protocols [1, 2, 3, 4, it came to be understood that quantum states can be employed for other cryptographic tasks, for example, for quantum secret sharing (QSS) of classical secrets [5]. A protocol for deterministic secure quantum communication (DSQC) using entangled photon pairs, wạs proposed by Shimizu and Imoto [6]. Although it was found to be insecure, it suggested that the prior generation of key (i.e. QKD) can be circumvented and protocols for unconditionally secure direct quantum communication of the message can De designed. Subsequently, many such protocols were proposed, which can broadly be divided into two classes: (a) those for quantum secure direct communication (QSDC) [7, 8, 9] and (b) those for DSQC [10, 11, 12, 13, 14, 15, 16, 17.

In DSQC receiver (Bob) can read out the secret message only after receipt of a pre-key: additional classical information of at least one bit for each qubit transmitted by the sender (Alice). By contrast, when no pre-key is required, a secure communication protocol is referred to as QSDC protocol [18]. A conventional QKD protocol generates the unconditionally secure key by quantum means but then uses classical cryptographic resources to encode the message. No such classical means are required in DSQC and QSDC. This interesting feature of DSQC and QSDC protocols has motivated several groups to study different aspects of DSQC and QSDC protocols in detail [[18] and reference therein].

QKD can be obtained from DSQC and QSDC protocols in the sense that given local resources like a random number generator, Alice can always implement QKD if she can implement DSQC or QSDC. Consequently, if we can establish that DSQC and/or QSDC protocol can be realized by using an arbitrary set of linearly independent quantum states, that would imply that QKD can also be realized using an arbitrary set of linearly independent quantum states. This is a motivation to study this type of protocols in the present paper.

The unconditional security of the existing protocols is claimed to be obtained by using different quantum resources. For example, unconditionally secure protocols are proposed a) with and without maximally entangled state [[11] and references therein], b) using teleportation [12] c) using entanglement swapping [13] d) using rearrangement of order of particles [15, 19] etc. Although based on very different resources, the security of all the existing protocols of secure direct quantum communication arises ultimately from the use of conjugate coding (i.e. from quantum non-commutativity). This is so because all the existing 
protocols of DSQC and QSDC detects Eve by measuring verification qubits in 2 or more mutually unbiased bases (MUBs). We thus classify all these protocols as essentially of BB84 type.

Now one may ask: Is conjugate coding essential for DSQC and QSDC? The answer is "no", as we have recently shown the same in a different context [20. Here we will first propose DSQC and QSDC protocols of BB84 type (i.e. one which uses conjugate coding) using arbitrary quantum states and will subsequently turn them to Goldenberg-Vaidman (GV) type [4 protocols, which uses only orthogonal states for encoding, decoding and error checking, as was done in the original GV protocol of QKD. So far the GV protocol has existed as an isolated orthogonal-state-based protocol of QKD. Our Bell-state-based generalization of original GV protocol may also be regarded as DSQC or QSDC, thus providing, in our opinion, the first instance of GV-type DSQC and QSDC protocols [20]. The idea is further extended here and it is shown that GV type protocols of DSQC and QSDC can be implemented using an arbitrary set of linearly independent quantum states.

In the protocols of DSQC and QSDC to be proposed in this paper, the rearrangement of orders of particles, plays a very crucial role. Therefore, it would be apt to note that the DSQC protocol based on the rearrangement of orders of particles was first proposed by Zhu et al. [15] in 2006. However, it turns to be insecure under a Trojan-horse attack, which can be corrected using the idea of rearrangement of particle order, as noted by Li et al. [11. The Li et al. protocol may thus be considered as the first unconditionally secure protocol of DSQC based on permutation of particles (PoP) .

In the last five years, many such PoP-based protocols of DSQC have been proposed. Very recently, Banerjee and Pathak 21], Shukla, Banerjee and Pathak [22, Yuan et al. [17] and Tsai et al. [19] have proposed PoP-based protocols for both DSQC and QSDC. The Yuan et al. protocol and Shukla-Banerjee-Pathak protocol use 4-qubit symmetric $W$ state for communication, while the Banerjee-Pathak protocol uses 3-qubit GHZ-like states, and the Tsai et al. protocol utilizes the dense coding of four-qubit cluster states.

Here we would also like to mention that in 2009 Xiu et al. 23] had provided a protocol of DSQC using the 5-qubit Brown state. Almost at the same time a DSQC protocol using the 5-qubit Brown state was also proposed by Jain, Muralidharan and Panigrahi [24]. Thus we observe that secure direct quantum communication, which was initially proposed using 2-qubit Bell states [7, 8] has subsequently been proposed with much more complex entangled states like 3-qubit GHZ-like state [21, 4-qubit $W$ state [17, 22], 4-qubit cluster state [19], and the 5-qubit Brown state [23, 24]. The widespread use of these maximally entangled states in DSQC, QSDC and QKD have by now established a common perception that these states are special and are important for the implementation of quantum cryptographic protocol.

Contrary to this common belief we will establish that DSQC and QSDC is possible with any arbitrary quantum state. Further, earlier proposed protocols are compared with each other and on the basis of qubit efficiency one is claimed to be better over the others. Here we aim to show that no quantum state is special and one can construct maximally efficient DSQC and QSDC protocol starting from any arbitrary set of linearly independent state vectors.

The remaining part of the present paper is organized as follows, in Section 2 we propose BB84-type protocols for DSQC and QSDC using arbitrary quantum state. In Section 3, we modify these protocols to corresponding orthogonal-state-based protocols of GV type. In Section 4 we analyze the security and efficiency of the proposed protocol and Section 5 is dedicated for the conclusions.

\section{The protocols of DSQC and QSDC}

Our aim is to show that efficient DSQC and QSDC protocols can be constructed using an arbitrary set of linearly independent quantum states. However, the information encoded states sent by Alice must be mutually orthogonal in order to enable Bob to unambiguously distinguish the encoded information. Let us assume that Alice starts with a set of $M \equiv 2^{n}$ arbitrary, linearly independent $n$-qubit state vectors $\left|x_{1}\right\rangle,\left|x_{2}\right\rangle,\left|x_{3}\right\rangle, \cdots,\left|x_{M}\right\rangle$.

If they are not pairwise orthogonal, Alice can apply Gram-Schmidt procedure on the above set of state vectors and obtain a set of mutually orthonormal state vectors as $\left\{\left|a_{1}\right\rangle,\left|a_{2}\right\rangle,\left|a_{3}\right\rangle, \cdots,\left|a_{M}\right\rangle\right\}$. This forms our arbitrary basis set. Let $\Pi_{J}(J=1,2, \cdots, M !)$ be an arbitrary permutation on $M$ letters, and let $\left\{\left|b_{j}\right\rangle \equiv\left|a_{\Pi(j)}\right\rangle\right\}$ represent the new basis obtained simply by permuting the states in the first basis. Effectively, we are relabeling the states $\left|a_{j}\right\rangle$. Now we may introduce the operators $U_{J}=\sum_{j}\left|b_{j}\right\rangle\left\langle a_{j}\right|$, which are unitary, as is easily verified to satisfy $U_{J} U_{J}^{\dagger}=U_{J}^{\dagger} U_{J}=\left(\sum_{p}\left|a_{p}\right\rangle\left\langle b_{p}\right|\right)\left(\sum_{q}\left|b_{q}\right\rangle\left\langle a_{q}\right|\right)=\left(\sum_{j}\left|a_{j}\right\rangle\left\langle a_{j}\right|\right)=\mathcal{I}_{M}$. In principle, if $\left\{\left|b_{j}\right\rangle\right\}$ is any basis set in $M$ dimension then $U_{J}$ will be unitary. However, for our purpose of showing that DSQC/QSDC is possible with an arbitrary set of mutually orthonormal state vectors $\left\{\left|a_{i}\right\rangle\right\}$ it is sufficient to consider $\left\{\left|b_{j}\right\rangle \equiv\left|a_{\Pi(j)}\right\rangle\right\}$. Further, this choice of output basis set is consistent with the spirit of the original GV protocol, where only single basis set is used (MUBs are not used) for encoding and decoding.

Thus $U_{J}$ are physically acceptable operators that can transform one state vector of our arbitrary basis set $\left\{\left|a_{j}\right\rangle\right\}$ into a state of the same basis set as basis set $\left\{\left|b_{j}\right\rangle \equiv\left|a_{\Pi(j)}\right\rangle\right\}$. This is physically expected as these operators are nothing but rotation in the state space spanned by the basis set $\left\{\left|a_{j}\right\rangle\right\}$. Now we may define an orthogonal family of $M$ unitaries $\left\{U_{j}\right\} \subset\left\{U_{J}\right\}$ as one that satisfies $\left\langle a_{i}\left|U_{j}^{\dagger} U_{k}\right| a_{i}\right\rangle=0$, where $\left|a_{i}\right\rangle \in\left\{\left|a_{j}\right\rangle\right\}$ would be used later as the initial state. Given a particular permutation of vectors $\left|b_{j}\right\rangle \equiv\left|a_{\Pi(j)}\right\rangle$, we construct our family of unitary operators $\left\{U_{j}\right\}$ as any set of $M$ operators that yield $\left|b_{j}\right\rangle=U_{j}\left|a_{i}\right\rangle$ and satisfy $\left\langle a_{i}\left|U_{j}^{\dagger} U_{k}\right| a_{i}\right\rangle=0$.

There are various alternative ways to construct $\left\{U_{j}\right\}$. For example, one particularly symmetric (in fact Hermitian) set $\left\{U_{j}\right\}$ 
for the case $\left\{\left|b_{j}\right\rangle \equiv\left|a_{j}\right\rangle\right\}$ is provided below as a specific example

$$
\begin{aligned}
U_{i} & =I, \\
U_{j \neq i} & =\left|a_{i}\right\rangle\left\langle a_{j}|+| a_{j}\right\rangle\left\langle a_{i}\left|+\sum_{k=1 ; k \neq i, j}^{M}\right| a_{k}\right\rangle\left\langle a_{k}\right| .
\end{aligned}
$$

Such operator families are used by Alice for encoding classical information.

It is worth observing here that what was noted above and the protocols below do not depend on whether the basis states $\left|a_{j}\right\rangle$ are single-particle or multi-particle states. The only requirement is that it is possible to split every state $\left|a_{j}\right\rangle$ into $n$ geometrically separable pieces that carry no information of the full state. If they are single-particle states, then $\left|a_{j}\right\rangle$ is an equal-weight superposition that lives in an $n$ (or greater) dimensional space, and constructed for $n$ spatially separated wave-packets.

Alice publicly announces the set of linearly independent states $\left\{\left|a_{j}\right\rangle\right\}$ to be used by her, the initial state $\left|a_{i}\right\rangle$ she is going to prepare and the set of unitary operators $\left\{U_{j}\right\}$ to be used by her for encoding. For the practical implementation purpose, our assumption is that Alice has devices to prepare states in the basis $\left\{\left|a_{j}\right\rangle\right\}$, to implement the set of unitary operators $\left\{U_{j}\right\}$, and that Bob has devices to make measurements in $\left\{\left|b_{j}\right\rangle\right\}$ basis. The main part of the protocol of DSQC works as follows.

\section{The DSQC protocol}

DSQC1: Alice prepares $\left|a_{i}\right\rangle^{\otimes N}$, which is an $N n$ qubit state (as $\left|a_{i}\right\rangle$ is $n$ qubit state). Qubits of $\left|a_{i}\right\rangle^{\otimes N}$ are indexed as $p_{1}, p_{2}, \cdots, p_{N n}$. Thus $p_{s}$ is the $s^{\text {th }}$ qubit of $\left|a_{i}\right\rangle^{\otimes N}$ and $\left\{p_{n l-n+1}, p_{n l-n+2}, \cdots, p_{n l}: l \leq N\right\}$ are the $n$ qubits of the $l^{\text {th }}$ copy of $\left|a_{i}\right\rangle$.

DSQC2: Alice encodes her $n$-bit classical secret message by applying one of the $n$-qubit unitaries $\left\{U_{j}\right\}=\left\{U_{1}, U_{2}, \cdots, U_{M}\right\}$. The encoding scheme, which is predefined and known to Bob, is such that $U_{1}, U_{2}, U_{3}, \cdots, U_{M}$ are used to encode $0_{1} 0_{2} \cdots 0_{n}, 0_{1} 0_{2} \cdots 1_{n}, 0_{1} 0_{2} \cdots 1_{n-1} 0_{n}, \cdots, 1_{1} 1_{2} \cdots 1_{n}$ respectively. Thus the coded states $U_{j}\left|a_{i}\right\rangle=\left|b_{j}\right\rangle$ are mutually orthogonal.

DSQC3: Using all the qubits of her possession, Alice creates an ordered sequence $P_{B}=\left[p_{1}, p_{2}, p_{3}, p_{4}, \cdots, p_{N n-1}, p_{N n}\right]$. She prepares $N n$ decoy qubits $11 d_{i}$ with $i=1,2, \cdots, n$ such that $d_{i} \in\{|0\rangle,|1\rangle,|+\rangle,|-\rangle\}$ and concatenates them with $P_{B}$ to yield a larger sequence $P_{B^{\prime}}=\left[p_{1}, p_{2}, p_{3}, p_{4}, \ldots, p_{N n-1}, p_{N n}, d_{1}, d_{2}, d_{3}, d_{4}, \ldots, d_{N n-1}, d_{N n}\right]$. Thereafter Alice applies a permutation operator $\Pi_{2 N n}$ on $P_{B^{\prime}}$ to create a random sequence $P_{B^{\prime \prime}}=\Pi_{2 N n} P_{B^{\prime}}$ and sends that to Bob. The actual order is known to Alice only.

DSQC4: After receiving Bob's authenticated acknowledgment of receipt of all the qubits, Alice announces $\Pi_{N n} \in \Pi_{2 N n}$, the coordinates of the decoy qubits. The BB84 subroutine to detect eavesdropping, is then implemented on the decoy qubits by measuring them in the non-orthogonal bases $\{|0\rangle,|1\rangle\}$ or $\{|+\rangle,|-\rangle\}$. If sufficiently few errors are found, then they go to the next step; else, they return to DSQC1.

All intercept resend attacks will be detected in this step and even if eavesdropping has happened Eve will not obtain any meaningful information about the encoding operation executed by Alice as the encoded sequence is rearranged.

DSQC5: Alice discloses the coordinates of the remaining qubits.

DSQC6: Bob measures his qubits in $\left\{\left|b_{j}\right\rangle\right\} \equiv\left\{\left|a_{\Pi(j)}\right\rangle\right\}$ basis and deterministically decodes the information encoded by Alice.

\subsection{Turning the DSQC protocol to a QSDC protocol}

The above protocol is clearly a protocol of DSQC as Alice needs to announce the actual order of the sequence. Rearrangement of particle ordering present in the DSQC protocol may be avoided by sending the information encoded states in $n$ steps and by checking eavesdropping after each step. To be precise, consider that Alice sends a sequence of all the first qubits first with $N$ decoy photons. If sufficiently few errors are found, only then does she send the sequence of all the second qubits, and so on. In such a situation the DSQC protocol presented above will be reduced to a QSDC protocol as no classical information will be required for decoding. Thus the previous protocol can be easily generalized to a QSDC protocol. To do so we need to modify DSQC3-DQSC5 in the above protocol. Therefore, the modified protocol may be described as follows:

\section{QSDC1: Same as DSQC1.}

\section{QSDC2: Same as DSQC2.}

QSDC3: Alice prepares $n$ sequences: $P_{B s}=\left[p_{s}, p_{s+n}, \cdots, p_{s+(N-1) n}: 1 \leq s \leq n\right]$. She also prepares $N n$ decoy qubits as in DSQC3 and inserts $N$ of the decoy qubits randomly into each of the $n$ sequences prepared by her. This creates $n$ extended sequences $\left(P_{B 1+N}, P_{B 2+N}, \cdots, P_{B n+N}\right)$ each of which contains $2 N$ qubits. Then she sends the first sequence $P_{B 1+N}$ to

\footnotetext{
${ }^{1}$ When $2 x$ qubits (a random mix of message qubits and decoy qubits) travel through a channel accessible to Eve and $x$ of them are tested for eavesdropping then for any $\delta>0$, the probability of obtaining less than $\delta n$ errors on the check qubits (decoy qubits), and more than $(\delta+\epsilon) n$ errors on the remaining $x$ qubits is asymptotically less than $\exp \left[-O\left(\epsilon^{2} x\right)\right]$ for large $x$. [25. As the unconditional security obtained in quantum cryptographic protocol relies on the fact that any attempt of Eavesdropping can be identified. Thus to obtain an unconditional security we always need to check half of the travel qubits for eavesdropping. This is why $N n$ decoy qubits are prepared. Decoy qubits can be prepared in any two MUBs.
} 
Bob. Receiving Bob's authenticated acknowledgment of receipt of $2 N$ qubits, she announces the positions of the decoy qubits in $P_{B 1+N}$. BB84 subroutine is then implemented on the decoy qubits to check eavesdropping and if sufficiently few errors are found then Alice sends $P_{B 2+N}$ to Bob and they check for eavesdropping and the process continues till the error free (i..e within the tolerance limit) transmission of $P_{B n+N}$. If at any stage of this step errors more than the tolerable rate is detected then they truncate the protocol and return to QSDC1 else they go to the next step.

QDDC4: Same as DSQC6.

Since Eve cannot obtain more than 1 qubit of a $n$-partite state (as we are sending the qubits one by one and checking for eavesdropping after each step) she has no information about the encoded state and consequently this direct quantum communication protocol is secure. Thus the rearrangement of particle order is not required if we do the communication in multiple steps. Further, this protocol does not require any classical communication for the decoding operation. Consequently, it is a QSDC protocol. Its qubit efficiency will be naturally higher than the previous protocol. This is so because here Alice does not need to disclose the actual sequence and consequently the amount of classical communication required for decoding of the message is reduced. But this increase in qubit efficiency is associated with a cost. This QSDC protocol will be slower compared to its DSQC counterpart as Alice has to communicate in multiple steps and has to check eavesdropping in each of the steps.

\section{Generalization to orthogonal state based protocols}

The security of the above protocols arises from the non-commutativity of the coding bases used in conjugate coding. However, GV protocol employs orthogonal code states, and does not require conjugate coding. In this section we will present a modified protocol that is independent of conjugate coding. Or in other words we will represent a GV type DSQC protocol using arbitrary states. In a completely orthogonal state based protocol like GV protocol both error checking and encoding are done by using orthogonal states. The DSQC and QSDC protocols presented above already employ orthogonal encoding; however, error checking is done by a BB84 subroutine, which uses non-orthogonal states (in fact, MUBs). To turn the proposed DSQC and QSDC protocols into corresponding GV-class protocols, which we call DSQC ${ }^{G V}$ and $\mathrm{QSDC}^{\mathrm{GV}}$, respectively, the error-checking in them also must employ only measurement in a single basis. In the following we will propose a new trick, which will allow us to implement the error checking using the Bell basis (alternatively by any other maximally entangled basis).

Intuitively, security in both the single-particle and multi-particle case arises because of no-cloning and the fact that Eve cannot access the full state at any given time. In Ref. [20], we pointed out that whereas quantum duality [26] is the fundamental origin of security in the GV protocol, monogamy of entanglement [27] turns out to be that of security in the multi-particle GV protocols obtained by us, suggesting, as a matter of considerable foundational significance, that duality and monogamy are two sides so of the same coin, a point to which we return later [28].

This unification is practically applied in the protocols presented in this section, which arises fundamentally from the observation that the security of the protocols we suggested above does not depend on whether the basis states $\left|a_{j}\right\rangle$ are single-particle or multi-particle states, as noted earlier. If $\left|a_{j}\right\rangle$ are taken as single-particle states, we obtain a higher dimensional equivalent of the GV protocol.

\subsection{Turning DSQC to DSQC ${ }^{\text {GV }}$}

As the encoding in the DSQC protocol is already done with the orthogonal states, so we retain the encoding steps (DSQC1 and DSQC2) and the decoding steps (DSQC5 and DSQC6) of the DSQC and modify error checking steps DSQC3 and DSQC4 as follows:

$\mathrm{DSQC}^{\mathrm{GV}}$ 3: Using all the qubits of her possession, Alice creates an ordered sequence $P_{B}=\left[p_{1}, p_{2}, p_{3}, p_{4}, \cdots, p_{N n-1}, p_{N n}\right]$. Now Alice prepares $\left[\frac{N n}{2}\right]$ Bell pairs $\left|\psi^{+}\right\rangle^{\otimes\left[\frac{N n}{2}\right]}$, where $\left|\psi^{+}\right\rangle=\frac{1}{\sqrt{2}}(|00\rangle+|11\rangle)$. She uses the $N n$ qubits of $\left|\psi^{+}\right\rangle^{\otimes\left[\frac{N n}{2}\right]}$ as decoy qubits and adds them with $P_{B}$ to yield a larger sequence $P_{B^{\prime}}=\left[p_{1}, p_{2}, p_{3}, p_{4}, \cdots, p_{N n-1}, p_{N n}, d_{1}, d_{2}, d_{3}, d_{4}, \cdots, d_{N n-1}, d_{N n}\right]$. Thereafter Alice applies a permutation operator $\Pi_{2 N n}$ on $P_{B^{\prime}}$ to create a random sequence $P_{B^{\prime \prime}}=\Pi_{2 N n} P_{B^{\prime}}$ and sends that to Bob. The actual order is known to Alice only.

DSQC $^{G V}$ 4: After receiving Bob's authenticated acknowledgment of receipt of all the qubits, Alice announces $\Pi_{N n} \in \Pi_{2 N n}$, the coordinates of the decoy qubits (Bell states). Bob measures the decoy qubits using Bell basis. If sufficiently few errors are found, then they go to the next step; else, they return to DSQC1.

\subsection{Turning QSDC to QSDC ${ }^{\mathrm{GV}}$}

To turn the QSDC into QSDC ${ }^{\mathrm{GV}}$ we just need to modify QSDC3 as follows (all the other steps will remain same):

QSDC $^{\mathrm{GV}}$ 3: Alice prepares $n$ sequences: $P_{B s}=\left[p_{s}, p_{s+n}, \cdots, p_{s+(N-1) n}: 1 \leq s \leq n\right]$. She also prepares $\left[\frac{N n}{2}\right]$ Bell pairs as in DSQC $^{G V} 3$ and inserts the qubits of the $\frac{N}{2}$ Bell pairs randomly into each of the $n$ sequences prepared by her. This creates $n$ extended sequences $\left(P_{B 1+N}, P_{B 2+N}, \cdots, P_{B n+N}\right)$ each of which contains $2 N$ qubits. Then she sends the first sequence $P_{B 1+N}$ to Bob. Receiving Bob's authenticated acknowledgment of receipt of $2 N$ qubits, she announces the positions of 
the decoy qubits in $P_{B 1+N}$. Bob measures the decoy qubits using Bell basis to check eavesdropping and if sufficiently few errors are found then Alice sends $P_{B 2+N}$ to Bob and they check for eavesdropping and the process continues till the error free (i.e. within the tolerance limit) transmission of $P_{B n+N}$. If at any stage of this step errors more than tolerable rate is detected then they truncate the protocol and return to $\mathbf{Q S D C}{ }^{\mathrm{GV}} \mathbf{1}$ else they go to the next step.

An interesting feature of these modified GV-type protocol is that the strict time checking required for the original GV protocol is not required here. This amplifies the experimental realizability of the proposed orthogonal state based protocols. Further, in the original GV protocol strictly one basis set is used for encoding, decoding and eavesdropping checking. Same is true for modified version of GV if $\left\{\left|a_{i}\right\rangle\right\}$ is Bell basis (or more precisely, if decoy sates are prepared in $\left\{\left|a_{i}\right\rangle\right\}$ basis). In all other cases encoding and decoding is done with a set of mutually orthogonal states and eavesdropping is checked with another set of orthogonal states. Extending this notion if we choose $\left\{\left|b_{j}\right\rangle \neq\left|a_{\Pi(j)}\right\rangle\right\}$ as an arbitrary output basis set then in the last step of the protocol Bob has to do measurement in this output basis and we would obtain GV-type protocol that uses three different sets of orthogonal basis sets. Thus the presented protocols may use two/three different basis sets to implement the protocol but they never use non-commutativity of those two/three basis sets to obtain the unconditional security of the protocol. This is so because eavesdropping is always checked with a single basis as required in GV type protocol. Origin of security in these protocols are explained below. In fact quantum mechanical origin of the security of these protocols distinguishes them from BB84 type of protocols.

\section{Efficiency and security of the protocol}

There are two aspects of the security of the DSQC and QSDC protocols: 1) We need to detect every possible attacks of Eve and 2) we need to detect Eve before she obtains any meaningful information. The first aspect is common in all secure quantum communication protocols (e.g. QKD). Now, since we are inserting adequate number of decoy qubits which are either prepared in a random sequence of $\{|0\rangle,|1\rangle,|+\rangle,|-\rangle\}$ in DSQC and QSDC schemes presented above, the eavesdropping checking process is equivalent to BB84 protocol and consequently we can detect all attacks of Eve. Similarly, when we inserts decoy qubits prepared in Bell basis then the eavesdropping checking process is equivalent to that in generalised GV protocol [20]. This step is sufficient for QKD because if the key is leaked to Eve then the key will not be used for encryption of any information in future but this is not sufficient for DSQC or QSDC because here we are directly sending information through the channel and we cannot afford to detect Eve after she has already got all the information.

As the communicated states are orthogonal states in principle Eve can intercept and resend them without being detected but the presence of decoy photon will detect Eve's attacks. On the other hand, if Eve attacks all qubits and measures them she will only obtain a random sequence of bits. PoP ensures that she will not obtain any meaningful information. Thus our protocol of DSQC with arbitrary states is unconditionally secure.

An important point here is that security is obtained in general by means of information splitting. First when we detect Eve, the entire state may be publicly available, but the positions of decoy photons are not announced till Bob receives all the photons. Without this information it is impossible for Eve to get the string on which information is encoded without being detected. But Eve would not mind being detected after she obtains the information by an intercept-resend attack (substituting dummies in place of real particles), because in that case she would have already obtained the encoded message.

Here PoP plays a crucial role, making the encoded information to be sent in essentially two or more pieces to Bob. Simultaneous non-availability of these information pieces makes it impossible for Eve to get any information. Thus the geographic information splitting plays an important role in the security of our protocol.

More precisely, for a given state $\left|b_{j}\right\rangle$, let the $M$ split pieces be denoted $|\alpha\rangle$, with $\alpha=1,2, \cdots, M$. Assume that $\left|b_{j}\right\rangle \in \mathcal{H}_{d}$, the $d$-dimensional Hilbert space of a single particle. Because of information splitting, the most general interaction between Alice's transmitted system and some probe with Eve is given by:

$$
\sum_{\alpha}|\alpha\rangle\langle\alpha| \otimes C_{\alpha}
$$

where $C_{\alpha}$ are operations acting only on the probe, prepared in an initial state $\left|0_{E}\right\rangle$. Intuitively, the more mutually distinguishable states $\left|\beta_{j}\right\rangle \equiv C_{j}\left|0_{E}\right\rangle$, the greater the entanglement generated between Alice's system and the probe, and thus more mixed is the system received by Bob. This is a manifestation of duality, which asserts a trade-off between the 'which-way' information Eve is effectively trying to get and the coherence observed by Bob 20. This disturbance, caused by Eve's restricted power in interaction with the communication system, forms the basis of security in GV. A rigorous derivation of duality in the qubit case, as applied to the security of GV with qubits, is given by us in Ref. 20] to be:

$$
\mathcal{P}+\mathcal{C} \leq 1
$$

where $\mathcal{P}$ and $\mathcal{C}$ denote which-way information and coherence, respectively.

In the multipartite case, a similar result holds, when we interpret $|\alpha\rangle$ in Eq. (2) to range over basis states of individual particles. The resulting entanglement with the probe causes a loss of coherence, which can be interpreted along the lines of Eq. (3). By virtue of the convexity of entanglement, this implies that the maximum entanglement or quantum correlation that 
the system can form with the rest of the universe is lesser than that which it could form if Eve's attack did not exist. More conventionally, as we clarify elsewhere [28], this can be expressed as the monogamy of entanglement

$$
E(A: C)+E(B: C) \leq E(A B: C),
$$

where $E(X: Y)$ is the square of concurrence, a measure of mixed-state entanglement [27].

The security of the extended GV-class protocols thus comes from duality or monogamy, which are understood to be facets of the same underlying phenomenon, which we have called monasticism [20, 28]. One reflection of this is that security checking requires the use of only a single basis, unlike the case of BB84-class protocols or the original QSDC or DSQC protocols, which require two or more mutually unbiased bases. In the original GV protocol, this basis is the $|0\rangle \pm|1\rangle$ basis, which can be generalized to an appropriate 'superposition' basis in the single-particle $d$-dimensional generalization of GV. In the bipartite generalization of GV (both in the QSDC or DSQC cases), this basis is typically that of the Bell states.

In the existing literature, two analogous but different parameters are used for analysis of efficiency of DSQC and QSDC protocols. The first one is simply defined as

$$
\eta_{1}=\frac{c}{q}
$$

where $c$ denotes the total number of transmitted classical bits (message bits) and $q$ denotes the total number of qubits used [14, 19. This simple measure does not include the classical communication that is required for decoding of information in a DSQC protocol. Consequently it is a weak measure.

Another measure [29] that is frequently used and which includes the classical communication is given as

$$
\eta_{2}=\frac{c}{q+b}
$$

where $b$ is the number of classical bits exchanged for decoding of the message (classical communication used for checking of eavesdropping is not counted). It is straightforward to visualize that $\eta_{1}=\eta_{2}$ for all QSDC and QSDCGV protocols but $\eta_{1}>\eta_{2}$ for all DSQC protocols.

Now in the proposed DSQC and DSQC ${ }^{\mathrm{GV}}$ protocols, $n$ bits of classical information are sent by $n$-qubits and equal number (i.e. $n$ ) of decoy qubits so we have $c=n$ and $q=2 n$. Further to disclose the actual order we need $n$ bits of classical information. Thus $b=n$. Therefore, for DSQC and DSQCGV protocols we have $\eta_{1}=\frac{1}{2}$ and $\eta_{2}=\frac{1}{3}$, and similarly for QSDC and QSDC ${ }^{G V}$ protocol we have $\eta_{1}=\eta_{2}=\frac{1}{2}$.

These are the upper bounds on the qubit efficiency of this kind of protocols, as shown in 21, 22. As the generalized protocols proposed here uses arbitrary quantum states to achieve these upper bounds, consequently, we may conclude that the efficiency of DSQC and QSDC protocols of present kind is independent of the choice of state. We have already shown that the security of the protocol is also independent of the choice of state. Consequently, all set of mutually orthogonal states are equivalent as far as secure quantum communication is concerned. There is no advantage of one state over the others and no essential advantage arises through new DSQC and QSDC protocols that use complex states like 5-qubit Brown state or 6-qubit cluster state.

\section{Conclusions}

It is shown that the DSQC and QSDC protocols of maximum efficiency can be constructed by using any arbitrary linearly independent set of quantum states applicable to single-particle or multi-particle cryptography. Generalized protocols for the same are proposed and their efficiency and security are analyzed. It is observed that the proposed protocols are unconditionally secure and maximally efficient for all states. Further, the protocols are generalized to completely orthogonal state based protocols. This interesting idea is expected to be of much use in all future experimental developments as it provides a wide choice of states to experimentalists. Whereas the security of conventional QSDC and DSQC protocols, like that of BB84-class QKD protocols, is based on quantum non-commutativity, the security of the GV versions of these protocols is based fundamentally on duality (in the single-particle case) or monogamy of entanglement (in the multi-particle case).

Acknowledgment: AP thanks Department of Science and Technology (DST), India for support provided through the DST project No. SR/S2/LOP-0012/2010 and he also thanks the Operational Program Education for Competitiveness - European Social Fund project CZ.1.07/2.3.00/20.0017 of the Ministry of Education, Youth and Sports of the Czech Republic.

\section{References}

[1] C. H. Bennett and G. Brassed, in Proceedings of the IEEE International Conference on Computers, Systems, and Signal Processing, Bangalore, p. 175 (1984).

[2] A.K. Ekert, Phys. Rev. Lett. 67, (1991) 661.

[3] C.H. Bennett, Phys. Rev. Lett. 68, (1992) 3121.

[4] L. Goldenberg and L. Vaidman, Phys. Rev. Lett. 75, (1995) 1239. 
[5] M. Hillery, V. Buzek and A. Bertaiume, Phys. Rev. A 59, (1999) 1829.

[6] K. Shimizu and N. Imoto, Phys. Rev. A 60, (1999), 157.

[7] G. L. Long and X. S. Liu, Phys. Rev. A 65 (2002) 032302.

[8] K. Bostrom and T. Felbinger, Phys. Rev. Lett. 89 (2002) 187902.

[9] M. Lucamarini and S. Mancini, Phys. Rev. Lett. 94 (2005) 140501.

[10] J. Liu et al., Chin. Phys. Lett. 23 (2006) 2652.

[11] X. H. Li et al., J. Korean Phys. Soc. 49 (2006) 1354.

[12] F. L. Yan and X. Zhang, Euro. Phys. J. B 41 (2004) 75.

[13] Z. X. Man, Z. J. Zhang and Y. Li, Chin. Phys. Lett. 22 (2005) 18.

[14] T. Hwang, C. C. Hwang and C W Tsai, Euro. Phys. J. D 61 (2011) 785.

[15] A. D. Zhu, Y. Xia, Q. B. Fan, and S. Zhang, Phys. Rev. A 73 (2006) 022338.

[16] H. J. Cao and H.S. Song, Chin. Phys. Lett. 23 (2006) 290.

[17] H. Yuan et al., Int. J. Theo. Phys. 50 (2011) 2403.

[18] G. Long, et al., Front. Phys. China, 2 (2007) 251.

[19] C.W. Tsai, C.R. Hsieh, and T. Hwang, Eur. Phys. J. D 61 (2011) 779.

[20] P. Yadav, R. Srikanth and A. Pathak, arXiv:1209.4304

[21] A. Banerjee and A. Pathak, Phys. Lett. A (2012) DOI:10.1016/j.physleta.2012.08.032.

[22] C. Shukla, A. Banerjee and A. Pathak, Int. J. Theo. Phys. (2012) DOI: 10.1007/s10773-012-1311-7.

[23] X. M. Xiu et al., Opt. Comm. 282 (2009) 333.

[24] S. Jain, S. Muralidharan and P. K. Panigrahi, Euro. Phys. Lett. 87 (2009) 600008.

[25] M. A. Nielsen and I. L. Chuang, Quantum Computation and Quantum Information, Cambridge University Press, New Delhi (2008) 589.

[26] B. G. Englert, Phys. Rev. Lett. 77 (1996) 2154.

[27] V. Coffman, J. Kundu, W. K. Wootters, Phys. Rev. A 61 (2000) 052306.

[28] R. Srikanth, A. Pathak and S. Banerjee, under preparation.

[29] A. Cabello, Phys. Rev. Lett. 85 (2000) 5635. 